\title{
Incidência de Pneumonia Associada à Ventilação Mecânica em Pacientes com TCE Submetidos a Craniotomia
}

\author{
Jaqueline Souza de Santana \\ Escola Bahiana de Medicina e Saúde Pública \\ Escola Bahiana de Medicina e Saúde Pública e \\ Helena França Correia dos Reis Universidade Federal da Bahia (Rua Comendador \\ Pereira da Silva 174/806, Brotas - 40 285-040 \\ Salvador-BA, lenafrancorreia@yahoo.com.br) \\ Mônica Lajana Oliveira Almeida \\ Hospital Geral do Estado da Bahia \\ Mário Ferreira da Silva \\ Hospital Geral do Estado da Bahia \\ Mário de Seixas Rocha \\ Escola Bahiana de Medicina e Saúde Pública \\ Análise do Manuscrito \\ Corpo Editorial da Bahiana \\ Tipo de Análise do Manuscrito \\ Peer Blind Review \\ Recebido em Mai/11 \\ Aprovado em Jun/11
}

\section{Resumo}

Introdução: 0 suporte ventilatório invasivo constitui um dispositivo terapêutico imprescindível em pacientes com Traumatismo Crânio Encefálico grave. Nas UTIs a Pneumonia Associada à Ventilação Mecânica (PAV) é a complicação pulmonar que mais comumente acomete os pacientes internados em uso de Ventilação Mecânica (VM). Objetivo: Verificar a incidência de PAV em pacientes com TCE submetidos à craniotomia, assim como a associação da PAV com o tempo de VM, tempo de permanência e mortalidade na UTI. Métodos: Estudo de coorte prospectivo, realizado em hospital de referência no atendimento e tratamento de pacientes politraumatizados. A população compreende pacientes internados na UTI com diagnóstico de TCE, submetidos à craniotomia, que fizeram uso de suporte ventilatório invasivo durante a internação. As variáveis de interesse do estudo foram: ocorrência de PAV; idade; sexo; tipo de acidente; causa da VM; tempo de VM; tempo de internação na UTI; mortalidade na UTI. Resultados: 0 estudo contou com 77 pacientes, e destes, $90,9 \%$ pertenciam ao sexo masculino. Os indivíduos jovens predominaram no estudo, com média de idade de 36,6 $\pm 13,7$ anos. Dos 77 pacientes, $20(26,0 \%)$ foram acometidos por PAV. Foi encontrada associação entre a PAV e o tempo prolongado de VM e tempo de permanência na UTI. Não foi encontrada associação da PAV com o óbito. Conclusão: A PAV foi infecção frequente em pacientes com TCE submetidos à craniotomia e esteve associada à maior duração da VM e maior tempo de permanência na UTI.

Palavras-Chave: Lombalgia, Dor lombar, Fisioterapia

\section{Incidence of Ventilator-Associated Pneumonia ECT in Patients with Undergoing Craniotomy}

\section{Abstract}

Introduction: The invasive ventilatory support is an essential therapeutic device in patients with severe brain injury trauma. In the ICU Ventilator-Associated Pneumonia (VAP) is a lung complication that most commonly affects patients admitted to the use of mechanical ventilation (MV). Objective: To determine the incidence of VAP in patients with TBI who 
underwent craniotomy, as well as the association of VAP with a time of MV, length of stay and ICU mortality. Methods: A prospective cohort study, conducted at a referral hospital in the care and treatment of polytraumatized patients. The population comprises patients admitted to the ICU with a diagnosis of TBI who underwent craniotomy, which made use of invasive ventilatory support during hospitalization. The variables of interest in the study were: occurrence of VAP, age, sex, type of accident because of the VM, duration of MV, duration of ICU stay, ICU mortality. Results: The study included 77 patients and of these $90.9 \%$ were male. The young individuals predominated in the study, mean age $36.6 \pm 13.7$ years. Of the 77 patients, $20(26.0 \%)$ were affected by EPI. Association was found between the VAP and prolonged MV time and ICU stay. There was no association of VAP with death. Conclusion: The VAP was common infection in patients with TBI who underwent craniotomy and was associated with longer duration of MV and longer ICU stay.

Keywords: Low back pain, low back pain, Physiotherapy

\section{La incidencia de neumonía asociada al ventilador TEC en pacientes sometidos a craneotomía con}

\section{Resumen}

Introducción: El soporte ventilatorio invasivo es un instrumento esencial terapéutica en pacientes con trauma grave lesión cerebral. En la UCI neumonía asociada a ventilación mecánica (NAVM) es una complicación pulmonar que afecta más comúnmente a los pacientes ingresados en el uso de ventilación mecánica (VM). Objetivo: Determinar la incidencia de NAV en los pacientes con TCE que se sometieron a una craneotomía, así como la asociación de VAP con un tiempo de MV, duración de la estancia y la mortalidad en la UCI. Métodos: Estudio prospectivo de cohorte, realizado en un hospital de referencia en el cuidado y el tratamiento de pacientes politraumatizados. La población está compuesta por pacientes ingresados en la UCI con diagnóstico de lesión cerebral que se sometieron a una craneotomía, que hizo uso de la asistencia respiratoria invasiva durante la hospitalización. Las variables de interés en el estudio fueron: aparición de la NAVM, edad, sexo, tipo de accidente a causa de la VM, la duración de la VM, la duración de la estancia en la UCI, la mortalidad en la UCI. Resultados: El estudio incluyó a 77 pacientes y de estos el 90,9\% eran hombres. Los individuos jóvenes predominaron en el estudio, con una edad media $36,6 \pm 13,7$ años. De los 77 pacientes, 20 $(26,0 \%)$ se vieron afectados por el PAI. Se encontró asociación entre la VAP y el tiempo de ventilación mecánica prolongada y estancia en la UCI. No se encontró asociación de la NAVM con la muerte. Conclusión: La VAP fue la infección común en pacientes con TCE que se sometieron a una craneotomía y se asoció con una mayor duración de la VM y una mayor estancia en la UCI.

Palabras clave: Dolor de espalda baja, dolor de espalda, fisioterapia

\section{Incidence de la pneumonie sous ventilation assistée ECT chez les patients subissant une craniotomie avec}

\section{Résumé}

Introduction: Le soutien ventilatoire invasive est un dispositif essentiel thérapeutiques chez les patients avec un traumatisme crânien blessures graves. Dans l'USI pneumonie sous ventilation assistée (PVA) est une complication pulmonaire qui affecte le plus souvent les patients admis à l'utilisation de la ventilation mécanique (VM). Objectif: Déterminer l'incidence de la PVA chez les patients traumatisés crâniens qui ont subi une craniotomie, ainsi que l'association de la VAP avec un temps de MV, la durée du séjour et la mortalité aux soins intensifs. Méthodes: Une étude de cohorte prospective, menée dans un hôpital de référence dans les soins et le traitement des patients polytraumatisés. La population comprend les patients admis aux soins intensifs avec un diagnostic de TCC qui ont subi une 
craniotomie, qui a fait usage d'assistance ventilatoire invasive pendant l'hospitalisation. Les variables d'intérêt dans l'étude étaient: survenue de VAP, âge, sexe, type d'accident en raison de la VM, la durée de VM, la durée du séjour aux soins intensifs, la mortalité aux soins intensifs. Résultats: L'étude a inclus 77 patients et de ces $90,9 \%$ étaient des hommes. Les jeunes individus ont prédominé dans l'étude, l'âge moyen $36,6 \pm 13,7$ ans. Sur les 77 patients, $20(26,0 \%)$ ont été affectés par le PEV. Association a été trouvée entre la VAP et prolongée de temps MT et séjour aux soins intensifs. Il n'y avait aucune association de VAP avec la mort. Conclusion: La VAP a été une infection fréquente chez les patients ayant subi une craniotomie TBI et était associé à une durée plus longue des MT et long séjour aux soins intensifs.

Mots-clés: douleurs lombaires, douleurs lombaires, de physiothérapie

\section{Introdução}

o traumatismo cranioencefálico (TCE) é definido como uma lesão de natureza não degenerativa ou congênita, causada por uma agressão direta ou iniciada por um processo de aceleração e desaceleração de alta energia do cérebro dentro do crânio1,2. Pode ser provocado por acidentes de trânsito, quedas, agressões físicas, perfuração por arma branca ou de fogo e atividades esportivas ${ }^{1,3}$.

O principal objetivo dos cuidados intensivos no paciente com TCE grave é a manutenção da pressão de perfusão cerebral adequada enquanto o cérebro se recupera do dano sofrido. Neste sentido, deve-se evitar a hipotensão arterial e a hipertensão intracraniana, sendo um dos métodos utilizados a craniotomia descompressiva, cirurgia que tem como principal finalidade a redução imediata da pressão intracraniana ${ }^{4}$.

O rebaixamento do nível de consciência em pacientes com TCE é o principal fator de risco para a broncoaspiração e posterior admissão na Unidade de Terapia Intensiva (UTI), que tem como objetivos detectar e tratar as complicações da lesão primária, proteger as vias aéreas e fornecer uma melhor condição para o retorno da função cerebral. Portanto, pacientes com problemas relacionados ao Sistema Nervoso Central (SNC) na maioria das vezes necessitam de assistência ventilatória por insuficiência respiratória aguda (IRpA). Dessa forma, o suporte ventilatório invasivo constitui um dispositivo terapêutico imprescindível em pacientes com TCE grave1,5. No entanto, sua utilização não é isenta de complicações ${ }^{6,7}$.

A necessidade de entubação endotraqueal em pacientes com comprometimento neurológico como o TCE, é um fator de risco importante para o desenvolvimento de complicações pulmonares, visto que tal suporte reduz os mecanismos de defesa das vias aéreas superiores até a árvore traqueobrônquica8. Nas UTIs a pneumonia associada à ventilação mecânica (PAV) é a complicação pulmonar que mais comumente acomete os pacientes internados ${ }^{9,10,11,12,13}$. 0 risco de ocorrência é de $1 \%$ a $3 \%$ para cada dia de permanência em ventilação mecânica e a incidência varia de $9 \%$ a $68 \%$, dependendo do método diagnóstico utilizado e da população estudada9 ${ }^{9}, 10$.

Além de ser responsável pelo aumento das taxas de letalidade hospitalar, tem sido demonstrado que a pneumonia prolonga o tempo de internação, a duração da ventilação mecâ-nica e leva a um aumento considerável nos cus $\neg$ tos do tratamento ${ }^{11,12}$. A identificação dos fatores predisponentes e dos pacientes de maior risco para aquisição de pneumonia deve ser o primeiro passo na implantação de um programa de vigilância, cujo objetivo é a diminuição do número de casos desta infecção ${ }^{12}$. A mortalidade por PAV é alta, variando de $40 \%$ a $80 \%$ o que torna crucial o seu reconhecimento, bem como a execução de medidas preventivas ${ }^{14}$. Diante disso, o objetivo do presente estudo é verificar a incidência de PAV em pacientes com TCE submetidos à craniotomia, assim como a associação da PAV com o tempo de VM, tempo de permanência e mortalidade na UTI. 


\section{Material \& Método}

\section{Delineamento do estudo}

Trata-se de um estudo de coorte prospectivo, de natureza descritiva e analítica, realizado na UTI de um hospital público da rede estadual, referência no atendimento e tratamento de pacientes politraumatizados.

\section{Casuística}

Foram estudados pacientes internados na UTI com diagnóstico de Traumatismo Crânio Encefálico, submetidos à craniotomia no período de Abril/2009 a Dezembro/2009 e que fizeram uso de suporte ventilatório invasivo durante a internação. Todos os pacientes estudados tinham idade igual ou superior a 18 anos, e não houve delimitação de sexo.

Foram excluídos do estudo pacientes que apresentaram traumatismo raquimedular, diagnóstico de PAV na admissão da UTI e suspeita de morte encefálica associados ao TCE.

\section{Protocolo do estudo e variáveis de interesse}

A coleta de dados foi realizada utilizando-se os registros dos prontuários dos pacientes, assim como a observação diária de informações a respeito de complicações pulmonares, exames radiográficos e exames laboratoriais. Para todos os pacientes inseridos na pesquisa foram abertas fichas, onde seu preenchimento foi realizado por dados primários, coletados através da observação do paciente no leito e dados secundários através da consulta aos prontuários. Todos os pacientes do estudo foram acompanhados a partir da admissão na UTI, no intuito de verificar a ocorrência ou não de PAV até a alta da UTI ou óbito.

A PAV foi definida clinicamente pelas pesquisadoras com auxílio do fisioterapeuta plantonista, sendo considerada com o aparecimento de infiltrado pulmonar novo ou progressivo à radiografia de tórax, associado a pelo menos dois dos seguintes sinais: secreção traqueal purulenta, febre (maior que $38^{\circ} \mathrm{C}$ ), leucopenia e leucocitose $8,9,10$.

As variáveis consideradas como potenciais variáveis de interesse do estudo foram: ocorrência de pneumonia associado à ventilação mecânica; idade (em anos); sexo (masculino/feminino); tipo de acidente (moto/ atropelo/ carro/ agressão física/ PAF/ FAB/ outros); causa da VM (depressão do SNC/ neuroproteção/ IRpA/ lesões associadas/ outras); tempo de ventilação mecânica invasiva (em dias); tempo de internação na UTI (em dias); mortalidade na UT́T.

\section{Análise estatística}

As variáveis categóricas (ocorrência de PAV, sexo, tipo de acidente, causa da VM, mortalidade na UTI) foram expressas em termos percentuais, e as variáveis numéricas (idade, tempo de ventilação mecânica invasiva e tempo de internação na UTI) foram expressas em medida de tendếncia central (média ou mediana) e sua variabilidade (desvio padrão ou intervalo interquartil). Para a análise da associação entre as variáveis selecionadas e a incidência de pneumonia associado à ventilação mecânica, foram utilizados o teste do quiquadrado para comparação das variáveis categóricas, e na sua impossibilidade o teste exato de Fischer. 0 teste $t$ de Student foi utilizado para comparar o tempo de VM e a idade dos pacientes com e sem PAV. Para comparar o tempo de permanência na UTI entre os pacientes com e sem PAV foi utilizado o teste de Mann-Whitney. Os dados coletados foram armazenados e analisados no programa Software SPSS, versão 14.0.

\section{Aspectos Éticos}

Este estudo foi aprovado pelo Comitê de Ética em Pesquisa da Fundação Bahiana para Desenvolvimento das Ciências. Os pacientes foram incluídos no estudo após seus responsáveis assinarem o Termo de Consentimento Livre e Esclarecido. 


\section{Resultados}

A amostra final do estudo foi composta por 77 pacientes, e destes, 70 pertenciam ao sexo masculino, representando $90,9 \%$. Houve predominância de indivíduos jovens, com média de idade de 36,6 $\pm 13,7$ anos. Em relação ao tipo de acidente, o motociclístico foi o mais frequente, com 23 casos $(29,9 \%)$. (Tabela 1$)$.

Dentre as causas da VM, a neuroproteção foi a mais frequente, representada por 49 $(63,6 \%)$ pacientes (Tabela 2). Tomando-se como parâmetro a Escala de Coma de Glasgow para avaliação do nível de consciência na admissão, observou-se uma mediana de 11 e intervalo interquartil de 8,0 a 13,0. 0 tempo de internamento na UTI teve uma mediana de 9 dias e um intervalo interquartil de 6,0 a 14,0. 0 tempo médio de VM foi de 7,4 dias com desvio padrão de 3,4 dias.

Dos 77 pacientes estudados, $20(26,0 \%)$ foram acometidos por pneumonia associada à ventilação mecânica (Tabela 2). 0 tempo de VM nos pacientes com PAV foi significativamente superior aos daqueles que não apresentaram PAV $(9,35 \pm 3,60$ dias vs $6,70 \pm 3,02$ dias; $p=0,002$ ). A mediana do tempo de permanência na UTI foi significativamente maior nos pacientes com PAV (14 e intervalo interquartil de 10,0 a 18,0 vs 8 e intervalo interquartil de $6,0$ a 11,$0 ; p<0,001)$.

Considerando o total de 77 pacientes, três $(3,9 \%)$ pacientes evoluíram para o óbito na UTI. A proporção de óbito na UTI não diferiu entre os indivíduos com e sem PAV (5\% vs 3,5\%; $p=1,0$; respectivamente), não sendo encontrada desse modo associação entre PAV e óbito.

Não foi encontrada associação significante entre a PAV e o sexo. Entre os homens, a incidência de PAV foi de $25,7 \%$ e entre as mulheres de $28,6 \%(p=1,0)$. Também não foram observadas diferenças ao se comparar a média de idades entre os pacientes com e sem PAV. A média de idade dos pacientes com PAV foi de $37,6 \pm 16,9$ e nos sem PAV $36,2 \pm 12,6(p=0,69)$.

\section{Discussão}

A maioria dos pacientes analisados pertencia ao sexo masculino e o tipo de acidente mais frequente foi o motociclístico. Segundo estudos, a predominância do sexo masculino nas pesquisas envolvendo acidentes de motocicleta é marcante e varia de 79\% a 96\%. Acredita-se que, o predomínio desse sexo nos acidentes de trânsito está relacionado a uma maior exposição, assim como maior utilização desse tipo de veículo2,15.

Em relação à idade, diversas pesquisas nacionais afirmam que cerca de $70 \%$ das vítimas de acidente de trânsito têm idades entre 10 e 39 anos, por conseguinte, pertencentes ao grupo de adolescentes e adultos jovens2,15. Dessa forma, os dados deste estudo estão de acordo com os achados na literatura.

A neuroproteção foi a causa da VM mais freqüente neste estudo, e pode-se dizer que essa predominância está relacionada às conseqüências do acidente motociclístico. De acordo com estudo, a região de cabeça e pescoço é a segunda região do corpo mais acometida nos acidentes de moto, tendo como principal causa o traumatismo crânio encefálico ${ }^{15}$.

A incidência de PAV encontrada neste estudo está dentro de uma larga margem encontrada na literatura, que varia de $8 \%$ a $68 \% 1,9,10,14$. Acredita-se que esta grande diferença na incidência de PAV, nos diferentes estudos, se dá, em parte, pela dificuldade de padronização das técnicas de análise e meios de diagnóstico ${ }^{8,14}$. Hoje não existe nenhum teste padrão ouro para o diagnóstico de PAV e nenhum método específico para a exclusão de infecção pulmonar em pacientes ventilados mecanicamente com febre e síndrome de disfunção orgânica múltipla ${ }^{12}$.

Neste estudo os pacientes com PAV apresentaram uma incidência de óbitos maior que os pacientes sem PAV, porém não houve uma diferença estatisticamente significativa. Desta forma, o presente estudo corrobora a maioria dos estudos em relação ao maior número de óbitos em pacientes ventilados mecanicamente com PAV, quando comparados aos sem PAV10,11,16. Porém encontrou-se também na literatura, um estudo que apresentou que 
pacientes com PAV, potencialmente mais graves, apresentaram uma mortalidade menor do que aqueles em VM sem PAV14. Segundo alguns estudos, a PAV não foi fator de risco para aumento da mortalidade, e sim a necessidade do uso da VM acarretou no aumento da letalidade $\mathrm{e}^{10,14}$.

Ao comparar o tempo de VM à ocorrência de PAV, observou-se que os pacientes com PAV possuíam um maior tempo de VM, sendo esta associação estatisticamente significativa no presente estudo. Segundo outros autores o tempo de VM elevado é um fator reconhecido e fortemente associado ao desenvolvimento da pneumonia associado à ventilação mecânica10.

Estudos trazem que dentre todas as infecções hospitalares, a pneumonia é a mais freqüente em pacientes internados nas UTI. Além de ser responsável pelas altas taxas de letalidade, a PAV aumenta a permanência dos pacientes tanto na UTI como no hospital, e também os custos com a internação ${ }^{10,11,12,13,14,16,17}$.

\section{Conclusão}

Pode-se dizer que estimar a incidência de PAV não é fácil, principalmente pela dificuldade de padronização das técnicas de análise e meios de diagnóstico. Porém, sabendose que a PAV está diretamente relacionada às altas taxas de mortalidade em hospitais e tendo conhecimento da influencia direta que ela possui com o tempo prolongado de internamento na UTI e no hospital, torna-se crucial o reconhecimento da sua incidência, bem como a busca de medidas preventivas para esta complicação pulmonar. Neste estudo, a PAV foi infecção freqüente em pacientes com TCE submetidos à craniotomia esteve associada à maior duração da VM e maior tempo de permanência na UTI.

\section{Referências}

1. ABREU, M O; \& ALMEIDA, M L. “Manuseio da ventilação mecânica no trauma cranioencefálico: hiperventilação e pressão positiva expiratória final”, Revista Brasileira de Terapia Intensiva, 21(1): 72-79. 2009.

2. SOUSA, R M C; REGIS, F C; \& KOIZUMI, M S. "Traumatismo crânio-encefálico: diferenças das vítimas pedestres e ocupantes de veículos a motor", Revista de Saúde Pública, 33(1): 85-94. 1999.

3. MELO, J R T; SILVA, R A; \& MOREIRA, E D J. "Características dos pacientes com trauma cranioencefálico na cidade do Salvador, Bahia, Brasil", Arquivos de Neuro-Psiquiatria, 62(3-A): 711715.2004.

4. FALEIRO, R M; et al. "Craniotomia descompressiva para tratamento precoce da hipertensão intracraniana traumática", Arquivos de Neuro-Psiquiatria, 63(2-B): 508-13. 2005.

5. SABACK, L M P; ALMEIDA, M L; \& ANDRADE, W. “Trauma Cranioencefálico e Síndrome do Desconforto Respiratório Agudo: Como Ventilar? Avaliação da Prática Clínica", Revista Brasileira de Terapia Intensiva, 19(1): 2007.

6. BECKMANN, U; \& GILLIES, D M. "Factors Associated With Reintubation in Intensive Care: An Analysis of Causes and Outcomes", Revista Chest, 120(2): 538-42. 2001.

7. SOARES, T R; et al. "Retirada do leito após a descontinuação da ventilação mecânica: há repercussão na mortalidade e no tempo de permanência na unidade de terapia intensiva?", Revista Brasileira de Terapia Intensiva, 22(1): p. 27-32. 2010.

8. ZEITOUN, S S; et al. "Incidência de Pneumonia associada à ventilação mecânica em pacientes submetidos à aspiração endotraqueal pelos sistemas aberto e fechado: estudo prospectivo - dados preliminares", Revista Latino-Americana de Enfermagem, 9(1): 46-52. 2001.

9. TEIXEIRA, P J. Z., et al. "Pneumonia associada à ventilação mecânica: impacto da multirresistência bacteriana na morbidade e mortalidade",Jornal Brasileiro de Pneumologia, 30(6): 540-48. 2004.

10. GUIMERÃES, M M Q; ROCCO, J R. "Prevalência e prognóstico dos pacientes com pneumonia associada à ventilação mecânica em um hospital universitário", Jornal Brasileiro de Pneumologia, 32(4): 339-46. 2006. 
11. CARRILHO, C M D M; et al. "Pneumonia Associada à Ventilação Mecânica em Unidade de Terapia Intensiva Cirúrgica. Revista Brasileira Terapia Intensiva, 18(1): 2006.

12. CARRILHO, C M D M. "Fatores associados ao risco de desenvolvimento de pneumonia hospitalar na Unidade de Terapia Intensiva do Hospital Universitário Regional do Norte do Paraná, Londrina, PR", Revista da Sociedade Brasileira de Medicina Tropical, 32(4): 455-56. 1999.

13. RELLO, J., et al. "International Conference for the Development of Consensus on the Diagnosis and Treatment of Ventilator-Associated Pneumonia", Revista Chest, 120(3): 2001.

14. SILVESTRINI, T L; \& CRUZ, C E R N. "Pneumonia Associada à Ventilação Mecânica em Centro de Tratamento Intensivo", Revista Brasileira Terapia Intensiva, 16(4): 2004.

15. SANTOS, A M R; et al. "Perfil das vítimas de trauma por acidente de moto atendidas em um serviço público de emergência", Cadernos de Saúde Pública, 24(8): 1927-38. 2008.

16. HEYLAND, D K; et al. "The Attributable Morbidity and Mortality of Ventilator-Associated Pneumonia in the Critically Ill Patient", American Journal of Respiratory and Critical Care Medicine, (159): 1999.

17. KOLLEF M, H. "What is Ventilator-Associated Pneumonia and Why is it Important?" Respiratory Care, 50(6): 2005 . 


\section{Apêndice}

Tabela 1

Características Demográficas dos Pacientes com TCE Submetidos à Craniotomia

\begin{tabular}{l|c|c}
\hline \multicolumn{1}{c|}{ Variáveis } & $\mathrm{N}(\%)$ & Média $\pm \mathrm{DP}$ \\
\hline Idade & & $36,6 \pm 13,7$ \\
\hline Sexo Masculino & $70(90,9)$ & \\
\hline Tipo de Acidente & & \\
\hline Motociclístico & $23(29,9)$ & \\
\hline Agressão Física & $15(19,5)$ & \\
\hline Atropelamento & $7(9,1)$ & \\
\hline PAF & $6(7,8)$ & \\
\hline Automobilístico & $3(3,9)$ & \\
\hline FAB & $2(2,6)$ & \\
\hline Outros & $21(27,3)$ & \\
\hline Total & $77(100)$ & \\
\hline
\end{tabular}

OBS: $\mathrm{N}$ = número de indivíduos; DP = Desvio Padrão; PAF = Perfuração de Arma de Fogo; FAB

$=$ Ferimento de Arma Branca

Tabela 2

Causas da VM e Ocorrência de PAV nos Pacientes com TCE Submetidos á Craniotomia

\begin{tabular}{l|c}
\hline \multicolumn{1}{c|}{ Variáveis } & N (\%) \\
\hline Causa da VM & $49(63,6)$ \\
\hline Neuroproteção & $27(35,1)$ \\
\hline Depressão do SNC & $1(1,3)$ \\
\hline Outras & $20(26)$ \\
\hline PAV & \\
\hline
\end{tabular}

OBS: N = número de indivíduos; VM = Ventilação Mecânica; SNC = Sistema Nervoso Central; $\mathrm{PAV}=$ Pneumonia Associada à Ventilação 